\title{
Star Formation \& Molecular Gas over Cosmic Time
}

\author{
E. Daddi ${ }^{1}$, M. T. Sargent ${ }^{1}$, M. Béthermin ${ }^{1}$ and G. Magdis ${ }^{2}$ \\ ${ }^{1}$ CEA Saclay, DSM/Irfu/Sérvice d'Astrophysique, Orme des Merisiers, F-91191 Gif-sur-Yvette \\ Cedex, France \\ ${ }^{2}$ Department of Physics, University of Oxford, Keble Road, Oxford OX1 3RH, UK
}

\begin{abstract}
Recent observations have revealed the existence of a 'main sequence' of star-forming galaxies out to high redshift. While the majority of star-forming galaxies are observed to be close to this relation between star formation rate (SFR) and stellar mass, a smaller subset of the population - so-called 'starbursts' - displays specific star-formation rates and star-formation efficiencies that exceed those of normal (main-sequence) galaxies by up to an order of magnitude. A large degree of homogeneity and similarity has been observed for the properties of the population of normal galaxies across a broad redshift range, including a narrow correlation between their CO luminosity (hence gas content) and IR luminosity and an almost invariable IR SED getting warmer with redshift, while starburst galaxies display systematically different properties. This can be used to devise a simple description of the evolution of the star-forming galaxy population since $z \sim 2$ and, with a higher degree of uncertainty, even further back in time, in a scheme that we dub two star formation mode framework (2-SFM). We show how this can successfully reproduce the shape of the IR luminosity function of galaxies as a function of redshifts, and the IR number counts. Furthermore, we can link the cosmic evolution of the sSFR of main-sequence galaxies to the evolution of the molecular fuel reservoir and to derive estimates of the molecular gas mass functions of star-forming galaxies that are based on their empirically measured gas properties rather than simulations or semi-analytical modelling. We also infer the evolution of the cosmic abundance of molecular gas and briefly discuss its expected observational signature by molecular line emission, the CO luminosity function.
\end{abstract}

Keywords. galaxies: evolution, galaxies: formation, galaxies: high-redshift

\section{Introduction}

Star-forming galaxies (SFGs) at both high and low redshift are a mixture of (1) 'normal' SFGs obeying a tight relation - the 'star-forming main sequence' - between star formation rate (SFR) and stellar mass $M_{\star}$ (e.g. Brinchmann et al. 2004; Salmi et al. 2012), and (2) 'starbursts' with a strong excess specific star formation rate (sSFR) compared to typical galaxies on the SFR- $M_{\star}$ main sequence.

A series of studies on the star-forming population have improved our understanding in that they not only characterised the systematic differences between normal galaxies and starbursts, but also revealed striking similarities within these sub-populations, both at fixed and at different redshifts: starbursting sources are more compact on average (e.g. Elbaz et al. 2011; Rujopakarn et al. 2011) than main-sequence galaxies which have a stellar structure that is well-described by exponential disks (e.g. Wuyts et al. 2011), they display infrared (IR) line deficits (e.g. PAHs or in the far-IR the [CII]-line; Elbaz et al. 2011; Graciá-Carpio et al. 2011), both of which are consistent with the scenario of a highly compressed, more efficient star formation as is also evidenced by the up to tenfold higher star formation efficiency (SFE) revealed by molecular gas measurements of distant SFGs (e.g. Daddi et al. 2010b; Genzel et al. 2010). 

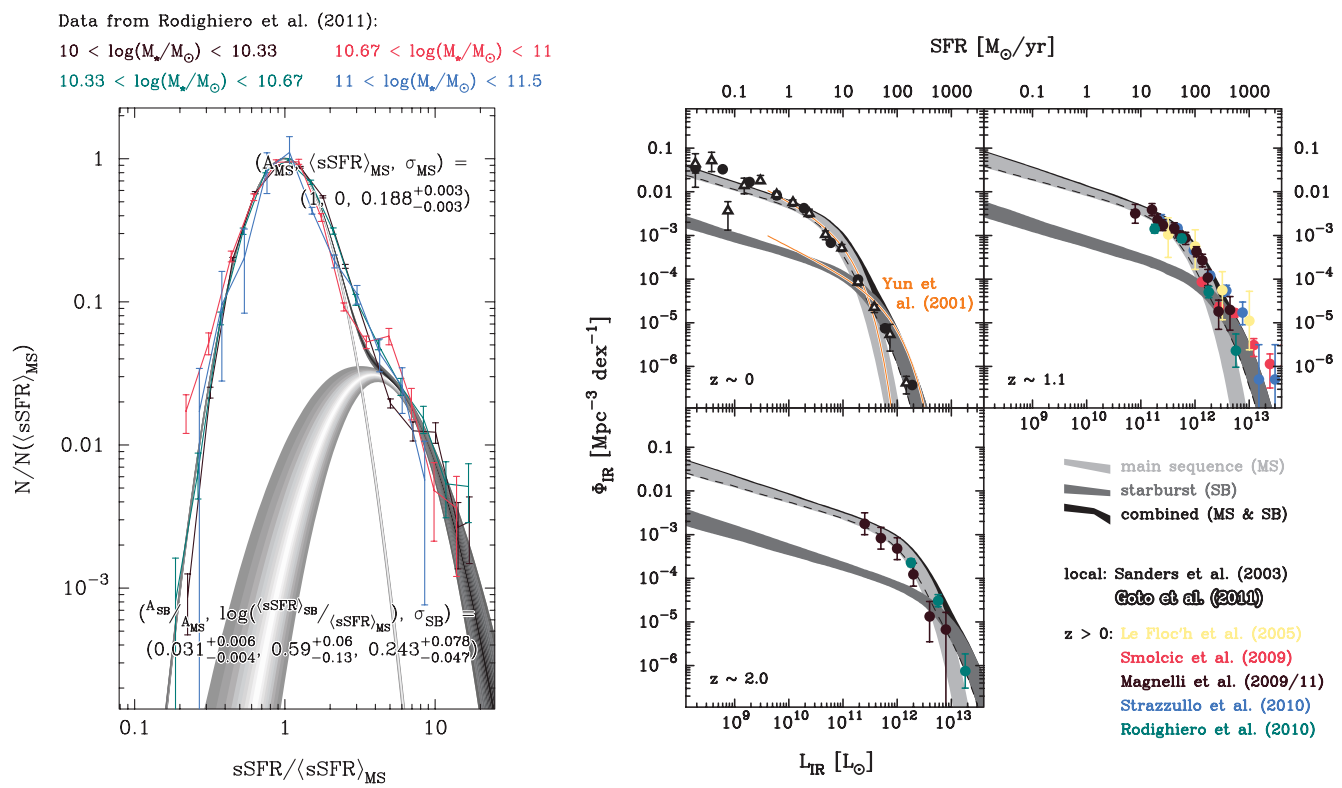

Figure 1. Left: Decomposition of the self-similar sSFR-distributions at $z \sim 2$ (four stellar mass bins with $M_{\star} / M_{\odot}>10^{10}$ from Rodighiero et al. (2011) are shown) into two log-normal contributions, of which one describes the distribution of main-sequence galaxies and the other one the distribution of enhanced (s)SFRs in galaxies displaying various degrees of starburstiness (likely due to interactions).

Right: Predicted contribution of normal (light grey) and burst-like (dark grey) star formation to IR LFs (SFR distributions; conversion between SFR and $L_{\mathrm{IR}}$ following Kennicutt (1998)) at $z<2$. Literature measurements are overlaid in colour; see legend.

In the following we will discuss a simple and self-consistent scheme (referred to as the '2 Star-Formation Mode framework'; 2-SFM) for the prediction of basic properties of sSFGs, such as the IR luminosity function (LF) and IR source counts or molecular gas mass functions. The 2-SFM framework relies on basic observables (e.g. the evolution of sSFR in main-sequence galaxies or their stellar mass distribution) and correlations between observables (e.g. the relation between stellar mass and SFR or the integrated Kennicutt-Schmidt relation linking their gas content and SFR). Its success thus provides a simple understanding of the star-forming galaxy population and its evolution with redshift.

\section{Infrared properties of star-forming galaxies: luminosity functions and source counts}

Distributions of $M_{\star}$ and SFR describe galaxy populations at a very basic level. The stellar mass function (MF) of SFGs is well-fitted by a Schechter function (e.g. Ilbert et al. 2010), whereas their IR LF (a proxy for the SFR distribution) is generally parametrized as a double exponential or power law function (e.g. Le Floc'h et al. 2005; Magnelli et al. 2009). A plausible explanation of this difference is the occurrence of burst-like and secular star formation activity among SFGs. The evolving shape of IR LFs hence implicitly contains information on the relative importance of the two modes of SF in the past. Here we discuss how the contribution of main-sequence and starburst galaxies to IR LFs (and thus to the SFR density) can be disentangled and use this information to reconstruct source counts from 24 to $1100 \mu \mathrm{m}$. 


\subsection{The IR luminosity function at $z \lesssim 2.5$}

The redshift evolution of the average sSFR in main-sequence galaxies out to $\mathrm{z} \sim 2$ (e.g. Elbaz et al. 2011, Karim et al. 2011) can be combined with the evolution of the stellar MF of SFGs to predict the shape of the IR LF if the distribution of sSFR at fixed stellar mass is known. Based on the results of Rodighiero et al. (2011) for SFGs at $z \sim 2$ we approximate this distribution by the double log-normal function on the left-hand side of Fig. 1 (identified as being due to main-sequence and - likely interaction-induced burst-like star formation activity, respectively; see Sargent et al. 2012 for details and a discussion of the underlying assumptions). The mapping of the stellar MF to an IR LF is effectively a convolution of the MF and a variable double-Gaussian kernel with (i) normalization fixed by the shape of the MF and (ii) main-sequence peak position that - given the redshift - is uniquely determined by the position of the SF main sequence in the (s)SFR vs. $M_{\star}$ plane. Thanks to our decomposition, we can then also identify the individual contribution of secular and burst-like SF activity to the IR LF (see Fig. 1, right).

In Fig. 1 we show that the evolution of the stellar MF and of the IR LF (i.e. of the SFR distribution) of star-forming galaxies at $\mathrm{z} \leqslant 2$ is self-consistent (see also Bell et al. 2007) and that starburst galaxies are the dominant factor shaping the bright end of the IR LFs. We find (cf. Sargent et al. 2012) that the fractional contribution of starburst activity to the cosmic SFR density (8-14\%) is only weakly redshift-dependent at $z<2$. We also reproduce the well-known fact that most local ULIRGs are starbursts (e.g., Sanders \& Mirabel 1996). At $z>0.9$ the majority of ULIRGs are main-sequence galaxies. Importantly, however, their high SFR $\left(>100 M_{\odot} / \mathrm{yr}\right)$ is not triggered by merging as in most local ULIRGs but is a secular process linked to large gas reservoirs in high- $z$ disks (e.g., Daddi et al. 2010a, Tacconi et al. 2010, Geach et al. 2011). Local and distant ULIRGs are intrinsically different objects for which direct comparisons should be avoided.

\subsection{IR source counts}

In addition to IR LFs, wavelength-dependent galaxy number counts are another important constraint for evolutionary models of IR-emitters. While purely semi-analytical models (e.g. Lacey et al. 2010) struggle to reproduce IR number counts, phenomenological or hybrid models (e.g. Béthermin et al. 2011; Gruppioni et al. 2011) fare better but are in general descriptive and use an evolution of the IR LF which is not physically motivated. However, these recent models - which reproduce the total counts passably - are excluded at $>3 \sigma$ by recent Herschel measurements of counts per redshift slice (Béthermin et al. 2012a). Here we present a new model of IR galaxy counts which builds on the 2-SFM framework introduced in Section 2.1.

Having derived SFR distributions (as measured by IR LFs, see Section 2.1) over a continuum of redshifts, the most important ingredient $\dagger$ for predicting IR source counts is the subsequent choice of the IR spectral energy distribution (SED) to assign to individual galaxies. Here we rely on a simple SED library based on Herschel observations: a single SED for the main sequence and another one for starbursts, both growing warmer with redshift (Magdis et al. 2012b). We compare the predictions of our model with measurements of differential galaxy counts from $24 \mu \mathrm{m}$ to $1.4 \mathrm{GHz}$ in Fig. 2. Overall, the observed

$\dagger$ Additional refinements, which are of lesser importance but which lead to an even better match of the IR source counts than is already achieved by the most basic, simplified prediction (cf. Béthermin et al. 2012b, and Fig. 2) are: a $M_{\star}$-dependent extinction law (e.g. Pannella et al. 2009), explicit modelling of an AGN component (significant $(>10 \%)$ only at $24 \mu \mathrm{m}$ at flux densities $>3$ mJy and negligible $(<2 \%)$ at longer wavelengths) and a correction for magnification caused by strong lensing. 

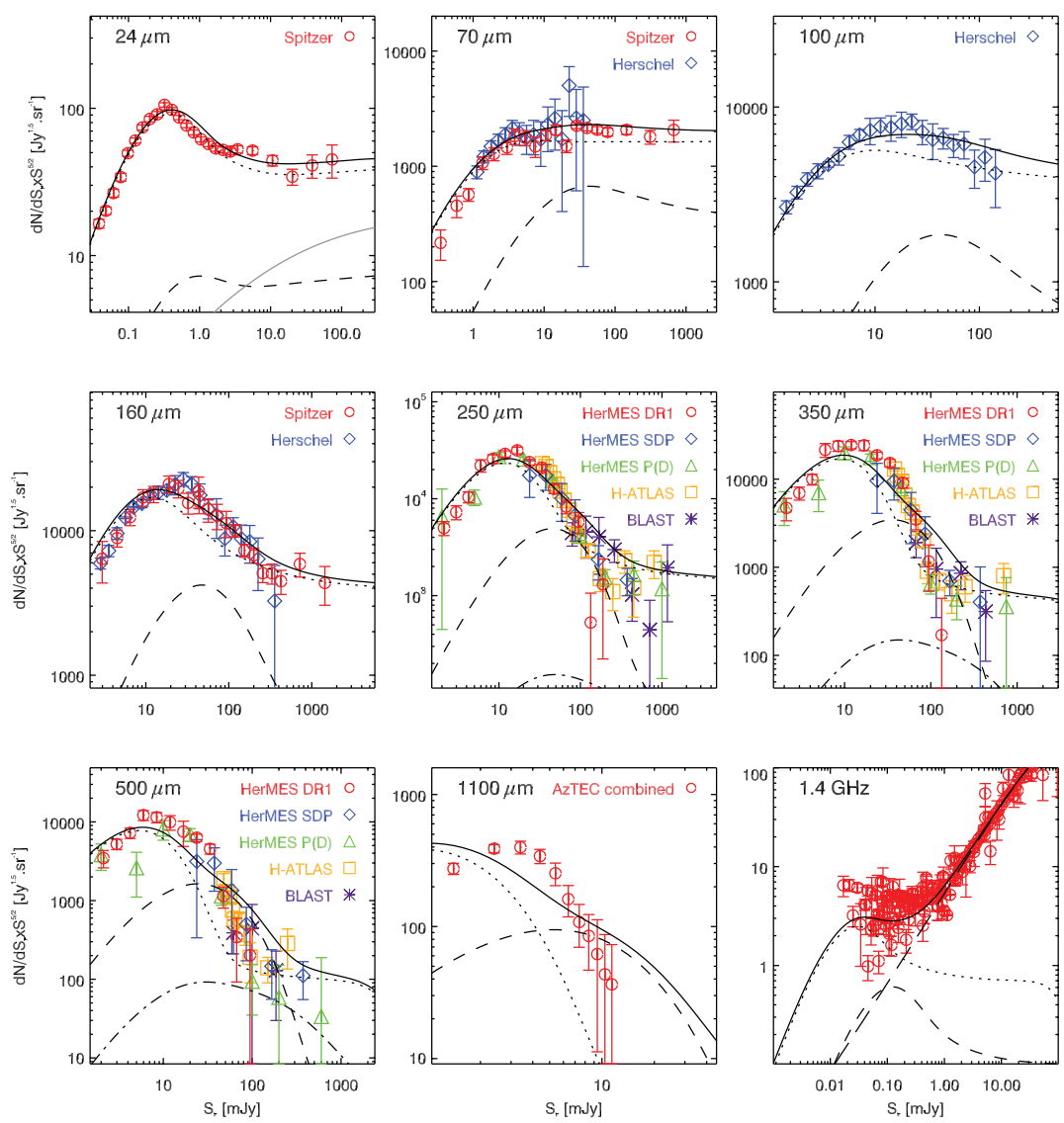

Figure 2. $24 \mu \mathrm{m}$ to $1.4 \mathrm{GHz}$ number counts (reproduced from Béthermin et al. 2012b). Solid line - total counts predicted by the 2-SFM framework, including a contribution for AGN-emission, mass-dependent dust attenuation and lensed sources; gray line - counts predicted when neglecting the three aforementioned refinements; dotted line - main-sequence contribution; short-dashed line - starburst contribution; dot-dashed line - lensed sources; triple-dot-dashed line - difference between counts with and without AGN contribution. At $1.4 \mathrm{GHz}$ our model for star-forming galaxies was combined with the model of AGN-driven radio sources by Massardi et al. (2010; long-dashed line).

source counts are well reproduced, showing the effectiveness of the core ingredient of the 2-SFM approach, namely the explicit distinction between normal and starbursting galaxies. By distinguishing between main-sequence and burst activity we can explore selection biases toward normal or starburst galaxies in surveys probing various wavelengths and flux density regimes. Main-sequence galaxies dominate the number counts at all flux densities and all wavelengths. However, the relative contribution of starbursts varies a lot with flux density and wavelength and is important $(\sim 30 \%)$ around $30 \mathrm{mJy}$ at $70 \mu \mathrm{m}$ and $50 \mathrm{mJy}$ at 350 and $500 \mu \mathrm{m}$.

Despite its simplicity, the 2-SFM predictions provide one of the best fits achieved so far to IR source counts, including counts per redshift slice at SPIRE wavelengths, which were poorly reproduced in earlier models. 

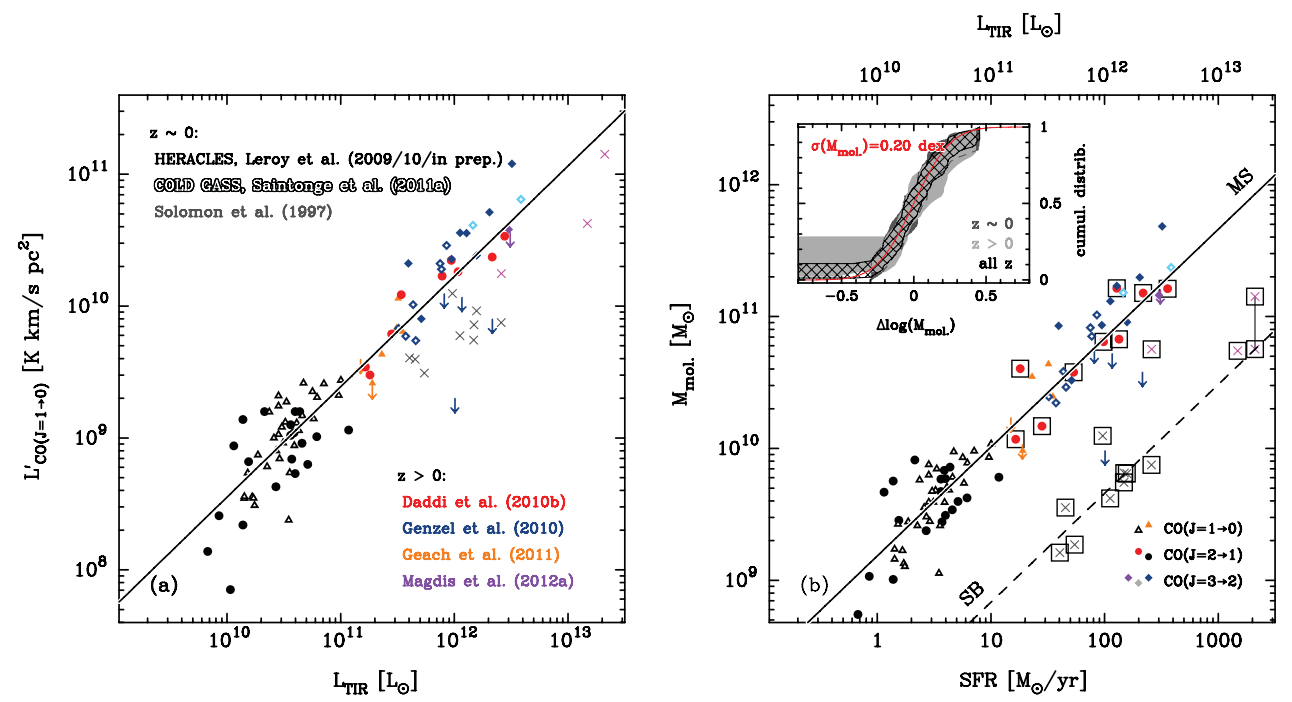

Figure 3. Correlation between measures of SFR and gas content for massive $\left(M_{\star}>10^{10} M_{\odot}\right)$, main-sequence galaxies in the nearby Universe and at $z \gtrsim 0.4$ (see legends in panel $(a)$ for data source). Selected starburst galaxies with measured conversion factors $\alpha_{\mathrm{CO}}$ from Solomon et al. (1997) and Magdis et al. (2012b) are plotted with crosses.

$(a)$ : Correlation between infrared luminosity $\left(L_{\mathrm{IR}}\right)$ and $\mathrm{CO}(1 \rightarrow 0)$ luminosity $\left(L_{\mathrm{CO}(1 \rightarrow 0)}^{\prime}\right.$; standard excitations corrections - e.g. Dannerbauer et al. (2009); Leroy et al. (2009) - were applied to $J>1$ transitions, cf. legend in panel $(b)$ ) with best-fitting relation derived for the main-sequence galaxy sample overplotted in black.

(b): Correlation between SFR and molecular gas mass $\left(M_{\text {mol. }}\right)$, the latter having been derived based on either (a) observational determinations of $\alpha_{\mathrm{CO}}$ (available for sources with boxed symbols) or (b) using a metallicity-dependent conversion factor (see text). The dispersion about the best-fit linear trend (solid black line) for main-sequence galaxies is approximately Gaussian with a dispersion $\sigma\left(M_{\mathrm{mol}}\right) \sim 0.2 \mathrm{dex}$ (red curve in inset). Dashed line - offset locus with approx. 15 -fold higher SFE for strong starburst galaxies.

\section{Molecular gas in star-forming galaxies near and far}

The well-constrained evolution at $z<2.5$ of the stellar MF and sSFR of SFGs and our decomposition of the sSFR distribution at fixed $M_{\star}$ into components due to normal and burst-like SFR activity provides a powerful framework for the prediction of cosmological observables, e.g. the evolution of molecular gas reservoirs in normal and starburst galaxies (see Section 3.3). As for the analytic-empirical modelling of the IR source counts in Section 2.2, assigning gas masses to galaxies based on their star-formation rate involves a population-dependent (i.e. one that varies depending on whether a source is undergoing burst-like or secular star-formation activity) choice of the star-formation efficiency (SFE). In Section 3.1 we discuss the typical behaviour of main-sequence and starburst galaxies with respect to their SFE and gas content and in Section 3.2 apply these scaling relations to show that the SSFR-evolution of main-sequence galaxies since $z \sim 3$ likely reflects the steady decrease of the gas fraction $\left(f_{\text {gas }}\right)$ in these sources over the same period.

\subsection{Gas reservoirs in star-forming galaxies: basic scaling relations}

CO follow-up studies of galaxies with stellar masses and SFRs representative of mainsequence galaxies have provided constraints on the molecular gas properties of roughly 50 published sources at $z \gtrsim 0.4$ over the past few years (e.g. Daddi et al. 2010a; Genzel et al. 2010; Tacconi et al. 2010; Geach et al. 2011). We combine this high- $z$ literature data with a roughly equally sized local sample - including massive galaxies from the 

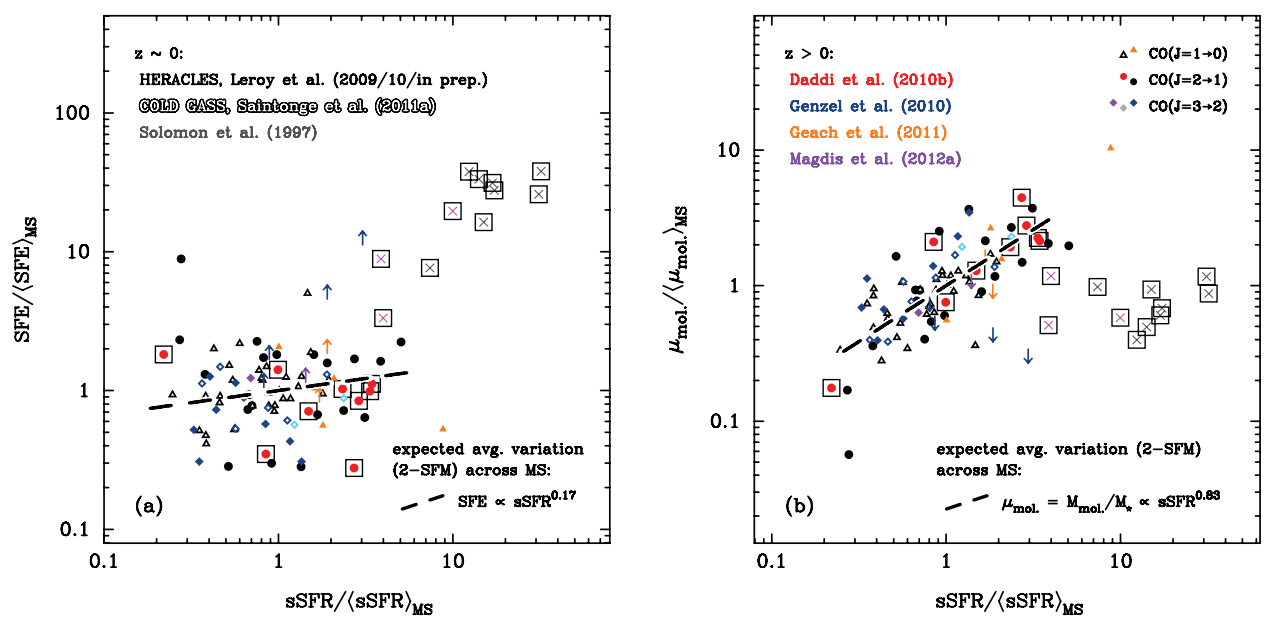

Figure 4. Star-formation efficiency (left), and molecular gas mass fraction, $\mu_{\text {mol. }} \equiv M_{\text {mol. }} / M_{\star}$ (right) vs. SSFR for selected main-sequence galaxies and starbursts at $z \lesssim 3$ (all measurements normalized to the $M_{\star}$ - and redshift-dependent average of the main-sequence and all symbols and data as in Fig. 3). When normalized to the characteristic main-sequence value, the homogeneous behaviour of main-sequence galaxies at all redshifts becomes visible: SFE varies very little within the main sequence while $f_{\text {gas }}$ steadily rises. Starbursting sources, on the other hand, have a gas content that is similar to the average main-sequence galaxy but display enhanced SFEs that lead to their excess (s)SFR.

HERACLES survey (Leroy et al. 2009) and AKARI-detected disks from the COLD GASS survey (Saintonge et al. 2011) - and plot their $L_{\mathrm{IR}}$ and CO-luminosity $L_{\mathrm{CO}}^{\prime}$ in Fig. 3 (left). The well-defined correlation between these observational proxies of SFR and molecular gas mass suggests that there is a high degree of homogeneity between low- and high-redshift main-sequence galaxies in terms of their molecular gas properties. We measure a dispersion of 0.2 dex in the correlation between molecular gas mass $M_{\text {mol }}$. and SFR (see Fig. 3, right), where we have applied either (a) observational determinations of $\alpha_{\mathrm{CO}}$ (available for sources with boxed symbols) or (b) a metallicity-dependent conversion factor $\dagger$ to convert CO-luminosities to molecular gas masses. The best-fit, inverse integrated Kennicutt-Schmidt (K-S) relation plotted in Fig. 3 is slightly sublinear $\left(M_{\mathrm{mol}} \propto \mathrm{SFR}^{\beta_{\mathrm{K}-\mathrm{S}}}\right.$, with $\left.\beta_{\mathrm{K}-\mathrm{S}}=0.83\right)$, but due to the small overlap in luminosity-space between local and $z>0$ main-sequence galaxies a redshift-dependent integrated K-S relation with different slope and changing normalisation currently cannot be excluded. Note, however, that by considering stacked samples of $z \sim 0$ and 2 main-sequence galaxies from Magdis et al. (2012b) - who inferred molecular gas masses indirectly by measuring average dust masses - the correlation is found to extend over at least two orders of (partially) overlapping magnitude in SFR at both redshifts, suggesting that the integrated $\mathrm{K}-\mathrm{S}$ relation to first order is a universal function of redshift for massive, normal SFGs $\left(M_{\star} / M_{\odot}>10^{10}\right)$ at $z \lesssim 3$.

To further highlight this uniform behaviour across a broad range of redshift, in Fig. 4 we plot SFE and gas fractions that have been normalized to the (mass- and

$\dagger$ Recent observational studies, as well as a numerical predictions on a metallicity-dependence of the conversion factor parametrized as $\alpha_{\mathrm{CO}} \propto Z^{\gamma}$ find a broad range of power-law exponents $\gamma$ ranging from -0.5 to -2 (e.g. Feldmann et al. 2012; Genzel et al. 2012; Schruba et al. 2012). Here we adopt an intermediate value of the exponent, $\gamma \simeq-0.9$, which results in the best fit to the $z \sim 0$ CO LF by Keres et al. (2003) (see Fig. 5) within the 2-SFM framework and its underlying assumptions (see Sargent et al. 2012, in prep.). 
redshift-dependent) average values of a galaxy located directly on the main-sequence and compare these to the analogously normalized sSFR (i.e. the offset from the main sequence). This representation of the data reveals that: (1) at all redshifts, the SFE in main-sequence galaxies in our "calibration sample" is almost independent of their position within the main sequence, while their SFR rises in lockstep with $f_{\text {gas }} ;(2)$ starburst galaxies (here we consider a small sample of local and high- $z$ starbursts with measured $\alpha_{\mathrm{CO}}$ ) have similarly-sized gas reservoirs as normal galaxies but are characterized by a strongly enhanced SFE. The dashed line in Fig. illustrates the expected variation of $\mathrm{SFE}\left(\propto \mathrm{sSFR}^{-0.17}\right)$ and $f_{\text {gas }}\left(\propto \mathrm{sSFR}^{0.83}\right)$ within the main sequence as it follows from the best-fit integrated K-S relation shown in Fig. 3.b, in agreement with the dust mass-based findings of Magdis et al. (2012b).

\subsection{Redshift evolution of gas fractions}

The well-defined relations between $M_{\star}$ and SFR, and between SFR and $\mathrm{H}_{2}$-mass allow for a straightforward prediction of the redshift-evolution of $f_{\text {gas }}$ in normal galaxies, where $M_{\text {gas }} / M_{\star} \equiv$ const. $\times \operatorname{SFR}\left(M_{\star}\right)^{\beta_{\mathrm{K}}-\mathrm{S}} / M_{\star}$ and $\operatorname{SFR}\left(M_{\star}\right)$ is given by the observed evolution of the sSFR of main-sequence galaxies. The gas fractions of normal galaxies at $z \lesssim 3$ predicted in this manner are in excellent agreement with literature data (Magdis et al. 2012a; but note that Narayanan et al. (2012) have recently questioned the veracity of the high measured $f_{\text {gas }}$ values based on their numerical simulations) suggesting that the gas content of secularly-evolving SFGs is an important driver of the cosmic sSFR-evolution. This is consistent with the observation in Fig. 4 that, also at fixed redshift, variations of sSFR across the main sequence are driven by varying $f_{\text {gas }}$.

\subsection{Molecular gas mass functions and the molecular gas history of the Universe}

To estimate the redshift evolution of the distribution of molecular gas masses in normal galaxies we convert the main-sequence component of the SFR-distribution depicted in Fig. 1 to a gas mass distribution by means of the best-fitting integrated K-S relation of Fig. 3.b. For the starbursting population we assume that the SFE increase (with respect to the value of the main-sequence state prior to the onset of burst activity) in these systems scales with the boost in star-formation rate engendered by the burst (see Sargent et al. 2012 for details). This kind of behaviour is suggested by the roughly similar excess in sSFR and SFE observed in Fig. 4.a and also seen in numerical simulations of galaxy mergers (e.g. Di Matteo et al. 2007). The total molecular gas MF (i.e. including both normal and starburst galaxies) inferred using this approach for the SFG population at $z \sim 0,2 \& 5$ is shown in Fig. 5 . Note that the $\mathrm{H}_{2}-\mathrm{MF}$ is only observationally constrained in the local Universe (Keres et al. 2003; Obreschkow \& Rawlings 2009a) where the measurements are entirely consistent with the 2-SFM predictions. (This is also true of the predicted CO LFs - cf. Fig. 5 - which follow from the $\mathrm{H}_{2}$-MFs after (a) assigning to main-sequence galaxies $\alpha_{\mathrm{CO}}$-values based on statistically-derived metallicities, computed using the fundamental metallicity relation of Mannucci et al. (2010) given the known SFR and $M_{\star}$, and (b) for starbursts assuming a conversion factor that scales inversely with the SFE-enhancement (see Sargent et al. 2012 in preparation for additional details and underlying assumptions/simplifications). The dominant contribution to the predicted $\mathrm{H}_{2}$-MF stems from main-sequence galaxies which hence also are the main contributors to the evolution of the cosmic abundance of molecular gas which is obtained by integration of the molecular gas mass functions and which is shown in Fig. 6. This indirect measurement of the comoving $\mathrm{H}_{2}$-density reveals a roughy 5 -fold increase of the molecular content of the Universe out to $z \sim 1.5$ where it roughly equals the stellar mass density and the cosmic abundance of atomic hydrogen. The increase traces the rise of 

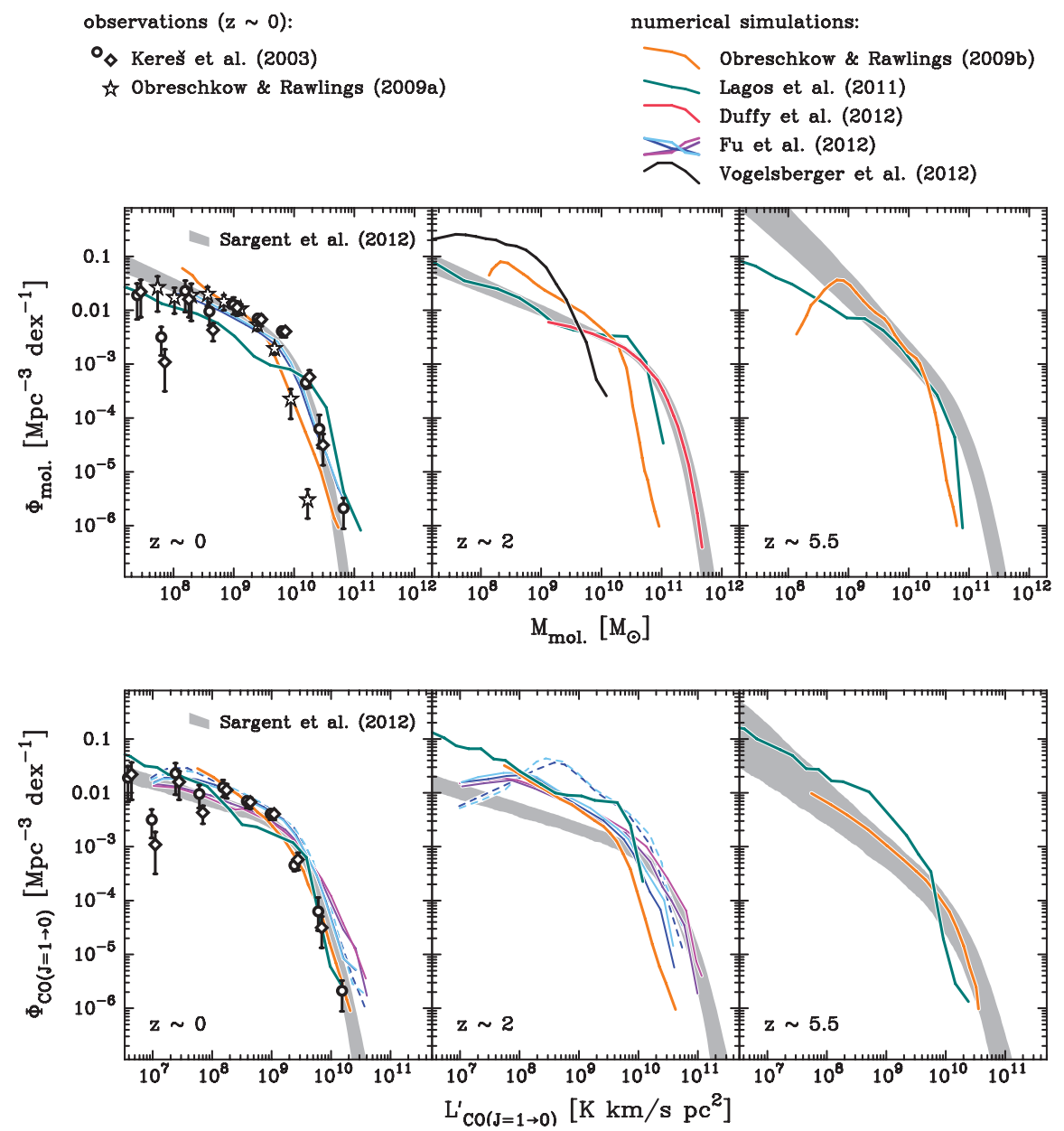

Figure 5. Molecular gas MFs (upper row; grey shading) and $\mathrm{CO}(1-0)$ LFs (lower row) based on (1) the evolution of the stellar MF of SFGs, (2) the redshift evolution of the sSFR of main-sequence galaxies, (3) the distribution of main-sequence and starbursting galaxies in the SFR- $M_{\star}$-plane (see Fig. 1, left), and (4) a metallicity-dependent conversion factor $\alpha_{\mathrm{CO}}$. MFs/LFs include contributions both from 'secular' and burst-like star formation (the latter being characterised by increased SFEs with respect to the secular mode which is assumed to be typical of galaxies residing on the main sequence). Coloured lines -predictions of recent semi-analytical models (see legend above figure) covering a similar redshift range as the empirically-motivated, predictive analysis of Sargent et al. (2012, in prep.) . At $z \sim 0$ we show observational constraints on the local MF/LF reported in Keres et al. (2003) and Obreschkow \& Rawlings (2009b).

the cosmic SFRD but is roughly five times smaller due to the sublinear relation between SFR and $\mathrm{H}_{2}$-mass found in Fig. 3 which implies slowly rising SFEs for the typical SFGs (of stellar mass $\sim 5 \times 10^{10} M_{\odot}$, cf. Karim et al. 2011) that contribute most to the cosmic SFRD density over this time.

\section{Summary}

Motivated by the homogeneity of the bulk of star-forming galaxies (in particular those occupying the so-called "main sequence" of star-forming galaxies) across a broad range of redshift we have presented a simple framework (2-SFM) for the prediction of 


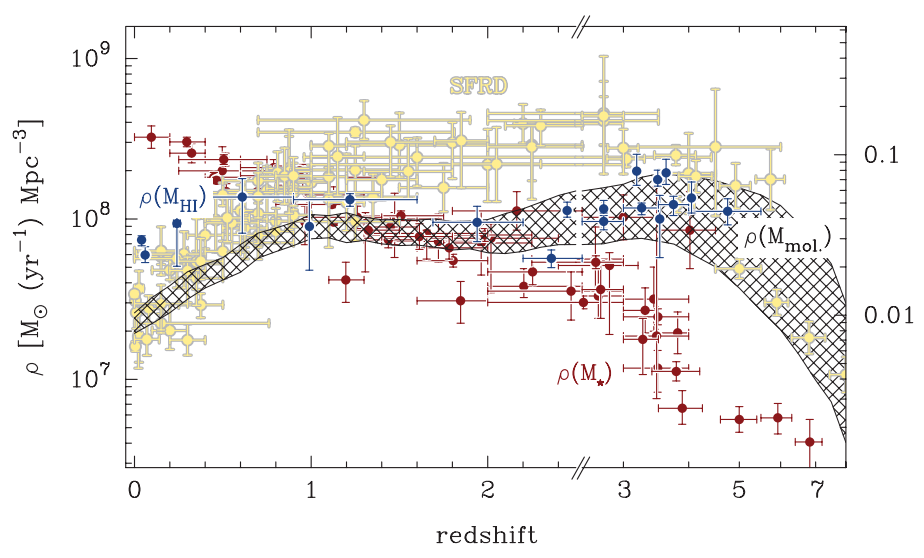

Figure 6. Redshift-evolution of the comoving mass density (cf. scale on left) of molecular gas, $\rho\left(M_{\mathrm{mol}}\right)$. The hatched region shows the evolution inferred from the integration of the predicted molecular gas MFs in Fig. 5 (extended to $z>2.5$ with a set of assumptions that successfully match high- $z$ stellar MFs and UV-LFs, e.g. González et al. 2010; Bouwens et al. 2011). The evolution of $\rho\left(M_{\mathrm{mol}}\right)$ is compared to compilations of the cosmic evolution of the atomic hydrogen abundance $\left(\rho\left(M_{\mathrm{HI}}\right)\right.$; e.g. Bauermeister et al. 2010, blue symbols) and of $\rho\left(M_{\star}\right)$, the stellar mass density (red symbols; see, e.g., compilation in Marchesini et al. 2009). Yellow symbols - SFRD-evolution (cf. scale on right), as constrained by literature data.

basic properties of the star-forming population. By explicitly distinguishing between (1) the population of 'normal' galaxies residing on the star-forming main sequence, and (2) starbursting galaxies for which we assume different characteristics (e.g. IR SEDs or a burst-dependent continuum of SFE enhancements) based on our currently best observational understanding, we show that this simple description is capable of reproducing the evolution of the IR LF out to $z \sim 2.5$ and IR/radio source counts at 24 to $1100 \mu \mathrm{m}$ and $1.4 \mathrm{GHz}$. We use the 2-SFM framework to predict the molecular gas mass function and $\mathrm{CO}(1 \rightarrow 0)$ luminosity function of star-forming galaxies, a fundamental observable which so far has only been measured at $z=0$ and the extension of which to higher redshift is a major goal of the ALMA era. The strong evolution of the inferred $\mathrm{H}_{2}$-mass function to higher masses with increasing redshift suggests that the cosmic $\mathrm{H}_{2}$-abundance was $\sim 5$ times larger at the peak of the cosmic SFH than in the local Universe. Furthermore, we provide strong evidence that the higher gas fractions in distant galaxies are directly reflected in the higher sSFR of these systems.

\section{References}

Bauermeister, A., Blitz, L., \& Ma, C.-P. 2010, ApJ, 717, 323

Bell, E. F., et al. 2007, ApJ, 663, 834

Béthermin, M., Dole, H., Lagache, G., Le Borgne, D., \& Penin, A. 2011, Aछ A, 529, 4

Béthermin, M., Le Floc'h, E., Ilbert, O., et al. 2012, A\&A A, 542, A58

Béthermin, M., Daddi, E., Magdis, G., et al. 2012, ApJ (Letters), 757, L23

Bouwens, R. J., Illingworth, G. D., Oesch, P. A., et al. 2011, ApJ, 737, 90

Brinchmann, J., Charlot, S., White, S. D. M., et al. 2004, MNRAS, 351, 1151

Daddi, E., et al. 2007, ApJ, 670, 156

Daddi, E., et al. 2010a, ApJ, 713, 686

Daddi, E., et al. 2010b, ApJ (Letters), 714, L118

Dannerbauer, H., Daddi, E., Riechers, D. A., et al. 2009, ApJ (Letters), 698, L178

Davé, R., Finlator, K., \& Oppenheimer, B. D. 2012, MNRAS, 421, 98

Di Matteo, P., Combes, F., Melchior, A.-L., \& Semelin, B. 2007, A\&A, 468, 61 
Duffy, A. R., Kay, S. T., Battye, R. A., et al. 2012, MNRAS, 420, 2799

Elbaz, D., et al. 2007, A\&SA, 468, 33

Elbaz, D., Dickinson, M., Hwang, H. S., et al. 2011, A\&A, 533, 119

Feldmann, R., Gnedin, N. Y., \& Kravtsov, A. V. 2012, ApJ, 747, 124

Fu, J., Kauffmann, G., Li, C., \& Guo, Q. 2012, MNRAS, 424, 2701

Geach, J. E., Smail, I., Moran, S. M., MacArthur, L. A., Lagos, C. d. P., \& Edge, A. C. 2011, ApJ (Letters), 730, L19

Genzel, R., et al. 2010, MNRAS, 407, 2091

Genzel, R., Tacconi, L. J., Combes, F., et al. 2012, ApJ, 746, 69

González, V., Labbé, I., Bouwens, R. J., et al. 2010, A\&A, 713, 115

Goto, T., et al. 2011, MNRAS, 414, 1903

Graciá-Carpio, J., Sturm, E., Hailey-Dunsheath, S., et al. 2011, ApJ (Letters), 728, L7

Gruppioni, C., Pozzi, F., Zamorani, G., \& Vignali, C. 2011, MNRAS, 416, 70

Ilbert, O., Salvato, M., Le Floc'h, E., et al. 2010, ApJ, 709, 644

Karim, A., et al. 2011, ApJ, 730, 61

Kennicutt, R. C., Jr. 1998, ARAA, 36, 189

Keres, D., Yun, M. S., \& Young, J. S. 2003, ApJ, 582, 659

Lacey, C. G., Baugh, C. M., Frenk, C. S., et al. 2010, MNRAS, 405, 2

Lagos, C.d.P., Baugh, C. M., Lacey, C. G., et al. 2011, MNRAS, 418, 1649

Le Floc'h, E., et al. 2005, ApJ, 632, 169

Leroy, A. K., et al. 2009, AJ, 137, 4670

Leroy, A. K., et al. 2011, ApJ, 737, 12

Magdis, G. E., Daddi, E., Sargent, M., et al. 2012, ApJ (Letters), 758, L9

Magdis, G. E., Daddi, E., Béthermin, M., et al. 2012, ApJ, 760, 6

Magnelli, B., Elbaz, D., Chary, R. R., et al. 2009, A\&̛A, 496, 57

Magnelli, B., Elbaz, D., Chary, R. R., et al. 2011, A\&GA, 528, A35

Mannucci, F., Cresci, G., Maiolino, R., Marconi, A., \& Gnerucci, A. 2010, MNRAS, 408, 2115

Marchesini, D., van Dokkum, P. G., Förster Schreiber, N. M., et al. 2009, ApJ, 701, 1765

Massardi, M., Bonaldi, A., Negrello, M., et al. 2010, MNRAS, 404, 532

Narayanan, D., Bothwell, M., \& Davé, R. 2012, MNRAS, 426, 1178

Obreschkow, D. \& Rawlings, S. 2009, MNRAS, 394, 1857

Obreschkow, D. \& Rawlings, S. 2009, ApJ (Letters), 696, L129

Pannella, M., Carilli, C. L., Daddi, E., et al. 2009, ApJ (Letters), 698, L116

Reddy, N. A., Pettini, M., Steidel, C. C., et al. 2012, ApJ, 754, 25

Rodighiero, G., et al. 2010, A\&A, 515, A8

Rodighiero, G., et al. 2011, ApJ (Letters), 739, L40

Rujopakarn, W., Rieke, G. H., Eisenstein, D. J., \& Juneau, S. 2011, ApJ, 726, 93

Saintonge, A., et al. 2011, MNRAS, 415, 32

Salmi, F., Daddi, E., Elbaz, D., et al. 2012, ApJ (Letters), 754, L14

Sanders, D. B., Mazzarella, J. M., Kim, D.-C., Surace, J. A., \& Soifer, B. T. 2003, AJ, 126, 1607

Sargent, M. T., Béthermin, M., Daddi, E., \& Elbaz, D. 2012, ApJ (Letters), 747, L31

Schruba, A., Leroy, A. K., Walter, F., et al. 2012, AJ, 143, 138

Smolčić, V., et al. 2009, ApJ, 690, 610

Solomon, P. M., Downes, D., Radford, S. J. E., \& Barrett, J. W. 1997, ApJ, 478, 144

Strazzullo, V., Pannella, M., Owen, F. N., et al. 2010, ApJ, 714, 1305

Tacconi, L. J., et al. 2010, Nature, 463, 781

Wuyts, S., Förster Schreiber, N. M., van der Wel, A., et al. 2011, ApJ, 742, 96 